Research Article

\title{
Event-Related Potentials Related to Anxiety in Emotion-Attention Interaction
}

\author{
Ssanghee Seo and Bonghyun Kim \\ Department of Computer Engineering, Kyungnam University, 11 Woryeongbuk 16-gil, \\ Masanhappo-gu, Changwon-si, Gyeongsangnam-do 631-701, Republic of Korea \\ Correspondence should be addressed to Bonghyun Kim; hyun1004@kyungnam.ac.kr
}

Received 28 August 2014; Accepted 23 September 2014; Published 8 October 2014

Academic Editor: James J. Park

Copyright (C) 2014 S. Seo and B. Kim. This is an open access article distributed under the Creative Commons Attribution License, which permits unrestricted use, distribution, and reproduction in any medium, provided the original work is properly cited.

\begin{abstract}
To identify neurological characteristics in accordance with anxiety under the interaction between emotion and attention, this study examines major ERP components when participants identify a target number by inhibiting task-irrelevant emotional face distractors. Experiments were conducted once per day at the same time for two days with 19 healthy adult men and women as required to study emotion-attention interaction. In this study, a variety of ERP components such as P100, N200, and P300 during experiment are significant. The amplitude and latency of the N200 component reflect both state and trait anxiety at all positions. This characteristic specially is prominently featured at Cz. Also, the latency of the late P300 component reflects the trait anxiety rather than state anxiety. The result of this study can help our understanding of the neurological responses related to anxiety during attentional control.
\end{abstract}

\section{Introduction}

Emotion and attention are essential elements in our everyday life and closely related to each other. Emotion may lead to attention and attention may cause emotion adversely [1]. Although studies of neurological activity related to emotion and neurological responses related to attention have been conducted separately, recent work suggests that emotion and attention are closely related and are involved in interdependent processing [2]. Generally, emotional stimuli command one's attention compared to different types of stimuli, with attentional bias by one's emotional valence. In real life, attention is maintained preferentially by a stimulus with emotional importance compared to a stimulus that is emotionally neutral. For stimuli specially that are potentially threatening, fear and negative emotions are prioritized. This attentional bias is a very normal response; however, individuals with higher anxiety levels more strongly perceive stimuli eliciting anxiety, and they remember these stimuli for longer times compared to individuals with lower anxiety levels [3]. Several studies have insisted that the attention systems of individuals with anxiety are more sensitive stimuli related to threats and are more biased $[4,5]$. These studies reported that anxiety disorder may show abnormal attentional bias to a wider range of stimuli and to specific stimuli. The attentional bias of patients with anxiety disorder appears to be faster and stronger [6]. Studies of the effects of anxiety on attentional control by inhibiting distractors analyzed behavior responses to compare cognition performance. Assessed were the saying of color names, reading text, eye movements, response times to specific stimuli, and error rates. Several major factors of emotion-attention appear within $500 \mathrm{msec}$ after a stimulus is presented. Thus, to understand the effects of anxiety on attentional control by inhibiting distractors, measurement data related to an early perception process is required, as are measurements of behavioral responses. The process of information processing of a specific stimulus in the brain can be observed directly because ERP (event-related potential) has high temporal resolution.

According to earlier work related to anxiety, patients with anxiety disorder show greater emotional confusion, attentional bias, and cognitive damage compared to people without such disorders [7-11]. These studies investigated ERP characteristics using IAPS images, emotional faces, and emotional words as the emotional stimuli presented. 
However, there are few studies of the effects of anxiety on the efficiency of attentional control. Few studies have examined the effects of anxiety on target identification while inhibiting emotional interfering stimuli to induce interaction between emotion and attention. Thus, the aim of this study is to detect the ERP components related to state and trait anxiety in an environment which requires target identification by inhibiting task-irrelevant emotional distractors, that is, interaction between emotion and attention. These neural characteristics can help us to understand the effects of anxiety on cognition processing and to determine therapy methods and therapy processes related to anxiety disorder.

\section{Methods}

2.1. Participants and Multisource Interference Task (MSIT). Nineteen right-handed graduates (10 males and 9 females; mean $=30$ years of age) from university participated in the study. All participants were free from psychiatric or neurological disorders. All participants provided written informed consent and were compensated for their participation. The study was approved by the Severance Hospital Ethics Review Board. Participants completed the Korean version of the State-Trait Anxiety Inventory (STAI; Spielberger et al. [12]) prior to every session. This study used a modified version of the Multisource Interference Task (MSIT) to induce emotionattention interaction [13]. All participants participated in two sessions for two days. This task presented three digits around the nose area of an emotional face. Pictures were selected from the FACES 3.3.1 database at the Max Planck Institute for Human Development. The pictures contained a set of images of six facial expressions: neutrality, sadness, disgust, fear, anger, and happiness. Also, the target stimuli consisted of three digits, two matching digits and one nonmatching digit. The one nonmatching digit was the target number. Participants were asked to press the target button using a number keypad, which was created in a laboratory. After taking a 10 min break with their eyes open, the task was presented for 3 minutes to familiarize the participants with the protocol prior to the start of the experiment. As illustrated in Figure 1, during the experiment, 144 fixation crosses and 144 pictures ( 6 facial types by 24 times) were each presented randomly for a period of $1.5 \mathrm{sec}$. For each participant, the response time to press the target button was recorded. Errors included incorrect key presses, missed key presses, or response times greater than $1500 \mathrm{~ms}$.

\subsection{Electroencephalography (EEG) Recording and Data Reduc-} tion. An EEG was continuously recorded for each participant during the task. The EEG was recorded with electrodes positioned according to the international 10-20 method of electrode placement, including the earlobes. The ground electrode was placed on the back of the neck (Iz), whereas the reference electrodes were placed on the right and left ears $(\mathrm{A} 1+\mathrm{A} 2)$. Eye movement artifacts were corrected using the ICA algorithm. All electrode impedances were less than $5 \mathrm{k} \Omega$. The EEG was amplified using a Biopac MP150 TM system, band-pass filtered $(0.1-100 \mathrm{~Hz})$, and digitized at a sampling

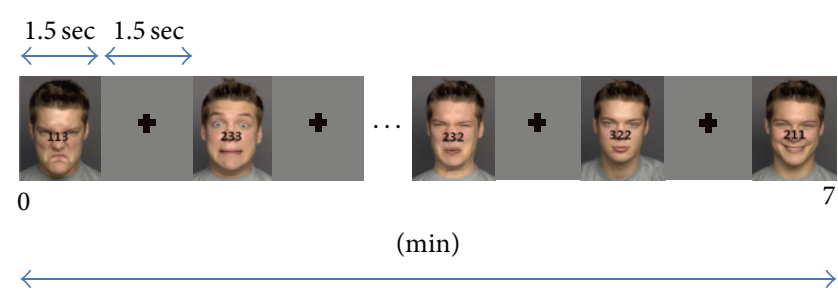

Total 144 images (6 types), 144 crosses

FIGURE 1: Schematic representation of the experiment design.

rate of $1000 \mathrm{~Hz}$. The high pass filter of the EEG signal was set to $0.5 \mathrm{~Hz}$, and the low pass filter was set to $100 \mathrm{~Hz}$. A $60 \mathrm{~Hz}$ notch filter was in use at all times. ERP amplitudes were obtained by stimulus-locked averaging from $0 \mathrm{~ms}$ to $1000 \mathrm{~ms}$ after baseline correction. Primary ERP analyses focused on the P100, N200, and P300 components during the task. The P100 (150-250 ms), N200 (250-350 ms), and P300 (350$450 \mathrm{~ms}$ ) components were compared by analyzing the mean amplitudes and peak latencies at the F3, F4, Cz, and $\mathrm{Pz}$ positions after stimulus onset.

To detect the correlation between anxiety and ERP components during MSIT, Pearson's correlation analyses between state/trait anxiety and the P100, N200, and P300 amplitudes and latencies were performed at each position (e.g., F3, F4, $\mathrm{Cz}$, and $\mathrm{Pz}$ ). Also, to test if there were effects of the face type used as a distractor, covariance analyses were performed on ERP components as a dependent variable. All statistical analyses were carried out using SPSS 14.0 (SPSS Inc., Chicago, IL).

\section{Results}

3.1. Interrelation between ERP and Anxiety. There were clearly ERP P100, N200, and P300 components at all positions during MSIT. To determine the correlation between the state/trait anxiety (STAI score) and the ERP component during MSIT, Pearson's correlation analyses were conducted. Table 1 shows the correlation scores between P100, N200, and P300 and state/trait anxiety by face type at each position. A minus symbol represents a nonsignificant difference. There were several findings, as follows. (1) The relationship between the N200 component and state/trait anxiety was significant at the F3, F4, and Cz positions. (2) The N200 amplitude at F4 was more closely linked with trait anxiety, whereas both state and trait anxiety were related to the N200 amplitude at F3 and Cz. Finally, (3) the higher the trait anxiety for a negative facial type, the slower the P300 latency at Pz.

3.2. Effect of Anxiety on ERP. We intended to identify whether ERP components were affected by state and/or trait anxiety during MSIT. Also, a covariance analysis was performed to detect if there is a difference in ERP components by face type used as distractor. The ERP P100, N200, and P300 amplitudes and latencies were used as dependent variables and six face types were used as fixed factors. Also, state and trait anxiety were used as covariates. As illustrated in Table 2, 
TABLE 1: Correlation scores among ERP components and anxiety.

\begin{tabular}{|c|c|c|c|c|}
\hline \multirow{2}{*}{ Position } & \multirow{2}{*}{ ERP components } & \multirow{2}{*}{ Facial type } & \multicolumn{2}{|c|}{ Correlation score (Sig.) } \\
\hline & & & State anxiety & Trait anxiety \\
\hline \multirow{9}{*}{ F3 } & \multirow{5}{*}{ N200 amplitude } & Disgust & $.526(.002)$ & $.626(.000)$ \\
\hline & & Fear & $.393(.029)$ & $.463(.009)$ \\
\hline & & Smile & $.459(.009)$ & $.430(.016)$ \\
\hline & & Neutral & $.392(.009)$ & $.389(.030)$ \\
\hline & & Sadness & $.447(.012)$ & - \\
\hline & \multirow{4}{*}{ N200 latency } & Annoy & $-.436(.014)$ & - \\
\hline & & Fear & $-.488(.005)$ & - \\
\hline & & Smile & $-.377(.036)$ & - \\
\hline & & Sadness & $-.401(.024)$ & - \\
\hline \multirow{8}{*}{$\mathrm{F} 4$} & \multirow{5}{*}{ N200 amplitude } & Annoy & - & $.385(.032)$ \\
\hline & & Disgust & $.480(.006)$ & $.598(.000)$ \\
\hline & & Fear & - & $.479(.006)$ \\
\hline & & Smile & $.443(.013)$ & $.435(.014)$ \\
\hline & & Neutral & - & $.393(.029)$ \\
\hline & \multirow{3}{*}{ N200 latency } & Fear & $-.499(.004)$ & - \\
\hline & & Smile & $-.369(.014)$ & - \\
\hline & & Sadness & $-.431(.016)$ & - \\
\hline \multirow{11}{*}{$\mathrm{Cz}$} & \multirow{6}{*}{ N200 amplitude } & Annoy & $.362(.045)$ & $.513(.003)$ \\
\hline & & Disgust & $.524(.002)$ & $.705(.000)$ \\
\hline & & Fear & $.449(.011)$ & $.548(.001)$ \\
\hline & & Smile & $.547(.001)$ & $.522(.003)$ \\
\hline & & Neutral & - & $.415(.020)$ \\
\hline & & Sadness & $.524(.002)$ & $.455(.010)$ \\
\hline & \multirow{5}{*}{ N200 latency } & Annoy & $-.402(.025)$ & - \\
\hline & & Fear & $-.449(.011)$ & - \\
\hline & & Smile & $-.404(.024)$ & - \\
\hline & & Neutral & $-.410(.022)$ & - \\
\hline & & Sadness & $-.436(.014)$ & - \\
\hline \multirow{3}{*}{$\mathrm{Pz}$} & \multirow{3}{*}{ P300 latency } & Annoy & - & $.369(.041)$ \\
\hline & & Disgust & - & $.367(.042)$ \\
\hline & & Fear & - & $.357(.049)$ \\
\hline
\end{tabular}

both state and trait anxiety had an effect on N200 amplitude and latency at all positions. Also, state anxiety influenced P300 latency at F3 and F4, and it influenced P100 latency at all positions. However, trait anxiety influenced P300 latency at all positions.

\section{Conclusions}

In this study, the effects of anxiety on cognitive functions were analyzed using ERP in a condition which required emotionattention interaction. The results can be summarized as follows. First, the P100 (early), N200 (middle), and P300 (late) components appear during the MSIT used in this paper. The tasks consisted of facial expressions as a distractor and three digits as targets. They required cognitive functions such as the sensory processing of facial expressions, target identification, and response decisions. Generally, it is known that the sensory processing of facial expressions is related to early ERP component, that target identification or the response decision is associated with the middle ERP component, and that the high-order cognitive function is related to the late ERP component. This result indicates that there are various ERP components related to emotion-attention interaction during MSIT. Second, the affected ERP amplitude and latency varied depending on the type of anxiety. The N200 amplitude becomes higher with higher state and trait anxiety. The P100 and N200 latencies become faster with higher levels of state anxiety. However, the P300 latency becomes slower with higher levels of trait anxiety. The P300 latency specially was closely related to trait anxiety at all positions. The present study suggests that specific ERP components are related to state anxiety or trait anxiety in an environment with contemporaneous emotional distractors and a target with which it is necessary to detect the target by inhibiting taskirrelevant distractors. Further, these findings may be used 
TABLE 2: Dependence between anxiety and ERP at position.

\begin{tabular}{|c|c|c|c|c|c|}
\hline Position & Dependent variable & Source & Mean square & $F$ & Sig. \\
\hline \multirow{7}{*}{ F3 } & P100 latency & State anxiety & .003 & 25.018 & .000 \\
\hline & N200 amplitude & State anxiety & .198 & 39.021 & .000 \\
\hline & N200 latency & State anxiety & .006 & 33.308 & .000 \\
\hline & N200 amplitude & Trait anxiety & .201 & 39.650 & .000 \\
\hline & N200 latency & Trait anxiety & .001 & 7.710 & .006 \\
\hline & P300 amplitude & Trait anxiety & .047 & 4.341 & .039 \\
\hline & P300 latency & Trait anxiety & .008 & 15.801 & .000 \\
\hline \multirow{9}{*}{$\mathrm{F} 4$} & P100 latency & State anxiety & .003 & 21.748 & .000 \\
\hline & N200 amplitude & State anxiety & .134 & 29.031 & .000 \\
\hline & N200 latency & State anxiety & .005 & 25.473 & .000 \\
\hline & P300 latency & State anxiety & .003 & 5.623 & .019 \\
\hline & P100 amplitude & Trait anxiety & .036 & 8.399 & .004 \\
\hline & N200 amplitude & Trait anxiety & .161 & 36.109 & .000 \\
\hline & N200 latency & Trait anxiety & .002 & 12.307 & .001 \\
\hline & P300 amplitude & Trait anxiety & .052 & 5.082 & .025 \\
\hline & P300 latency & Trait anxiety & .010 & 19.356 & .000 \\
\hline \multirow{10}{*}{$\mathrm{Cz}$} & P100 amplitude & State anxiety & .012 & 6.614 & .011 \\
\hline & P100 latency & State anxiety & .002 & 21.041 & .000 \\
\hline & N200 amplitude & State anxiety & .120 & 47.154 & .000 \\
\hline & N200 latency & State anxiety & .006 & 33.838 & .000 \\
\hline & P300 latency & State anxiety & .003 & 4.417 & .037 \\
\hline & P100 amplitude & Trait anxiety & .026 & 14.397 & .000 \\
\hline & N200 amplitude & Trait anxiety & .159 & 68.625 & .000 \\
\hline & N200 latency & Trait anxiety & .002 & 8.320 & .004 \\
\hline & P300 amplitude & Trait anxiety & .039 & 3.628 & .058 \\
\hline & P300 latency & Trait anxiety & .011 & 18.711 & .000 \\
\hline \multirow{7}{*}{$\mathrm{Pz}$} & P100 latency & State anxiety & .003 & 19.097 & .000 \\
\hline & N200 amplitude & State anxiety & .032 & 8.107 & .005 \\
\hline & N200 latency & State anxiety & .005 & 23.365 & .000 \\
\hline & N200 amplitude & Trait anxiety & .029 & 7.148 & .008 \\
\hline & N200 latency & Trait anxiety & .003 & 11.859 & .001 \\
\hline & P300 amplitude & Trait anxiety & .032 & 3.878 & .050 \\
\hline & P300 latency & Trait anxiety & .025 & 22.155 & .000 \\
\hline
\end{tabular}

as an index to assess cognition performance for clinical and nonclinical anxiety disorders.

\section{Conflict of Interests}

The authors declare that there is no conflict of interests regarding the publication of this paper.

\section{References}

[1] E. Ferneyhough, M. K. Kim, E. A. Phelps, and M. Carrasco, "Anxiety modulates the effects of emotion and attention on early vision," Cognition and Emotion, vol. 27, no. 1, pp. 166-176, 2013.

[2] L. Carretié, M. Martín-Loeches, J. A. Hinojosa, and F. Mercado, "Emotion and attention interaction studied through eventrelated potentials," Journal of Cognitive Neuroscience, vol. 13, no. 8, pp. 1109-1128, 2001.
[3] R. J. Compton, "The interface between emotion and attention: a review of evidence from psychology and neuroscience," Behavioral and Cognitive Neuroscience Reviews, vol. 2, no. 2, pp. 115-129, 2003.

[4] C. MacLeod and A. Mathews, "Anxiety and the allocation of attention to threat," The Quarterly Journal of Experimental Psychology, vol. 40, no. 4, pp. 653-670, 1988.

[5] A. Mathews and C. MacLeod, "Induced processing biases have causal effects on anxiety," Cognition and Emotion, vol. 16, no. 3, pp. 331-354, 2002.

[6] Y. Bar-Haim, D. Lamy, L. Pergamin, M. J. BakermansKranenburg, and M. H. van Ijzendoorn, "Threat-related attentional bias in anxious and nonanxious individuals: a metaanalytic study," Psychological Bulletin, vol. 133, no. 1, pp. 1-24, 2007.

[7] N. Derakshan, T. L. Ansari, M. Hansard, L. Shoker, and M. W. Eysenck, "Anxiety, inhibition, efficiency, and effectiveness: an investigation using the Antisaccade task," Experimental Psychology, vol. 56, no. 1, pp. 48-55, 2009. 
[8] M. W. Eysenck and M. G. Calvo, "Anxiety and performance: the processing efficiency theory," Cognition and Emotion, vol. 16, pp. 409-434, 1992.

[9] C. Georgios, "A comparison between experienced and inexperienced video game players perceptions," Human-Centric Computing and Information Sciences, vol. 3, article 15, 2013.

[10] G. M. Nagi, R. Rahmat, F. Khalid, and M. Taufik, "Regionbased facial expression recognition in still images," Journal of Information Processing Systems, vol. 9, no. 1, pp. 173-188, 2013.

[11] B. Debotosh, "Adaptive polar transform and fusion for human face image processing and evaluation," Human-Centric Computing and Information Sciences, vol. 4, article 4, 2014.

[12] C. D. Spielberger, R. L. Gorsuch, and R. E. Lushene, Manual for the State-Trait Anxiety Inventory, Consulting Psychologists Press, Palo Alto, Calif, USA, 1970.

[13] N. C. Ebner, M. Riediger, and U. Lindenberger, "FACES-a database of facial expressions in young, middle-aged, and older women and men: development and validation," Behavior Research Methods, vol. 42, no. 1, pp. 351-362, 2010. 

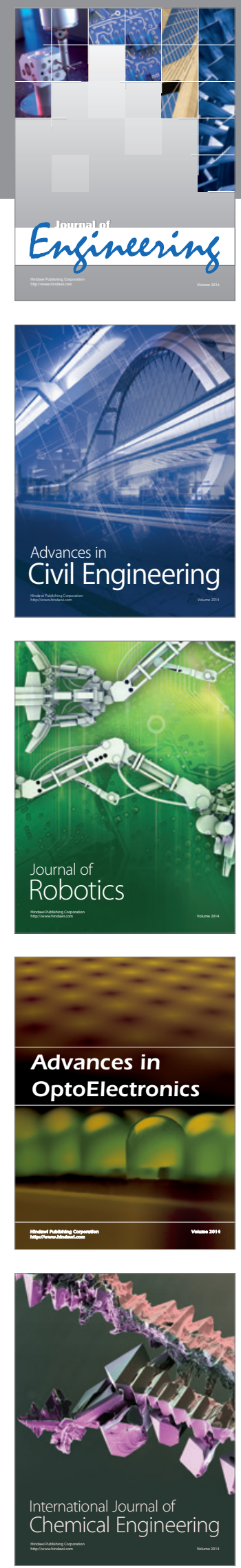

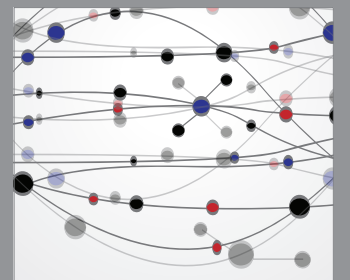

The Scientific World Journal
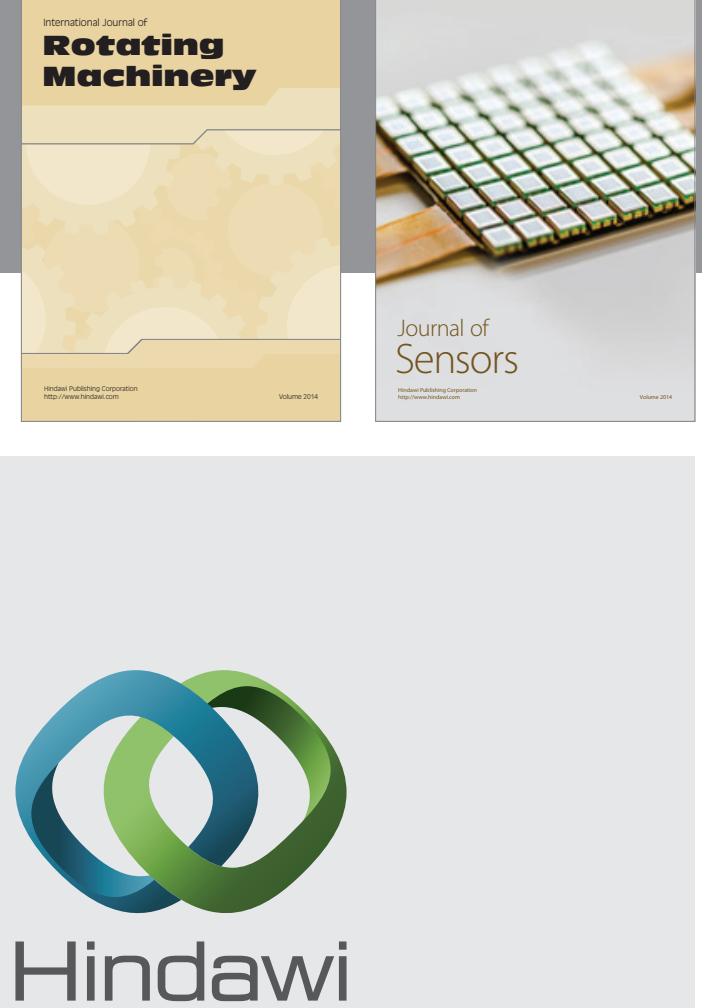

Submit your manuscripts at http://www.hindawi.com
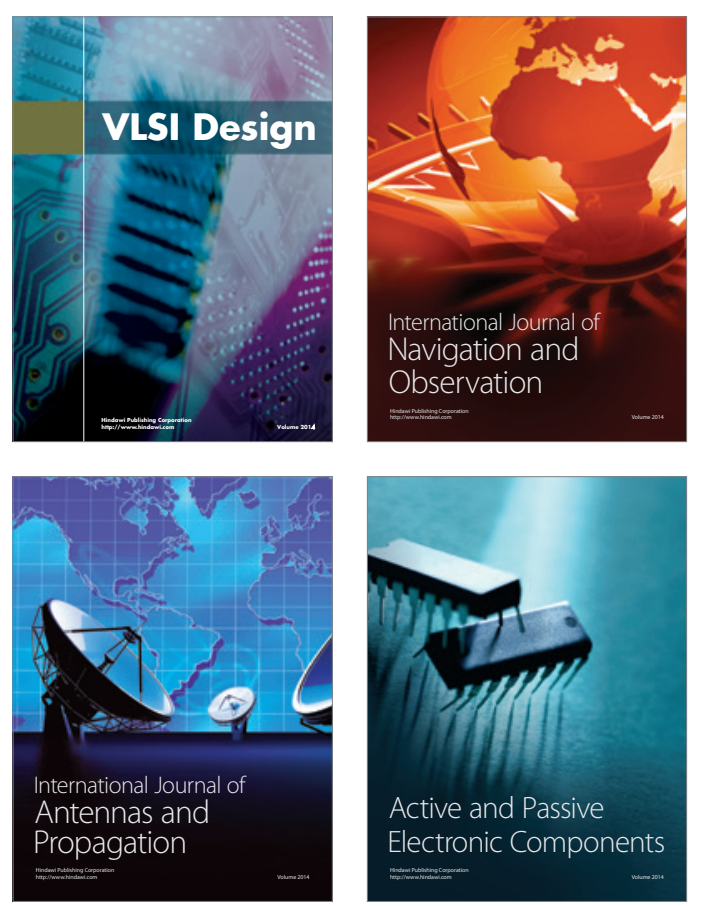
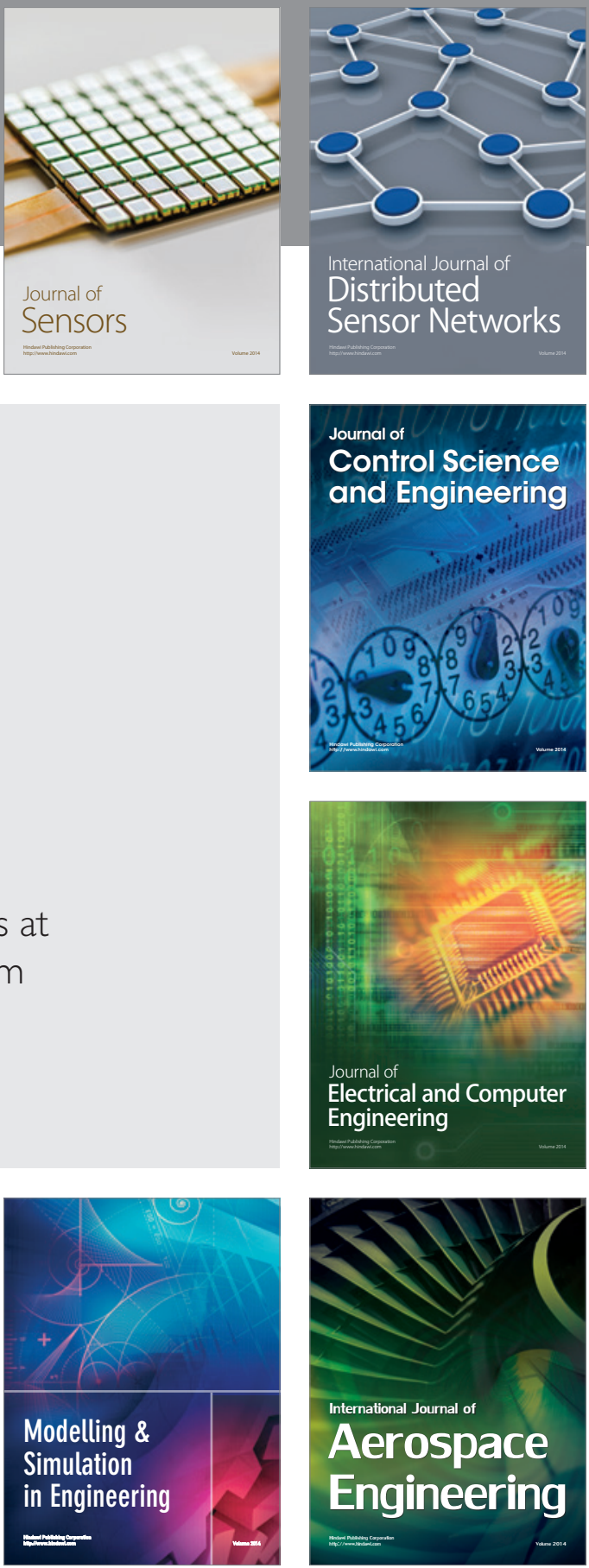

Journal of

Control Science

and Engineering
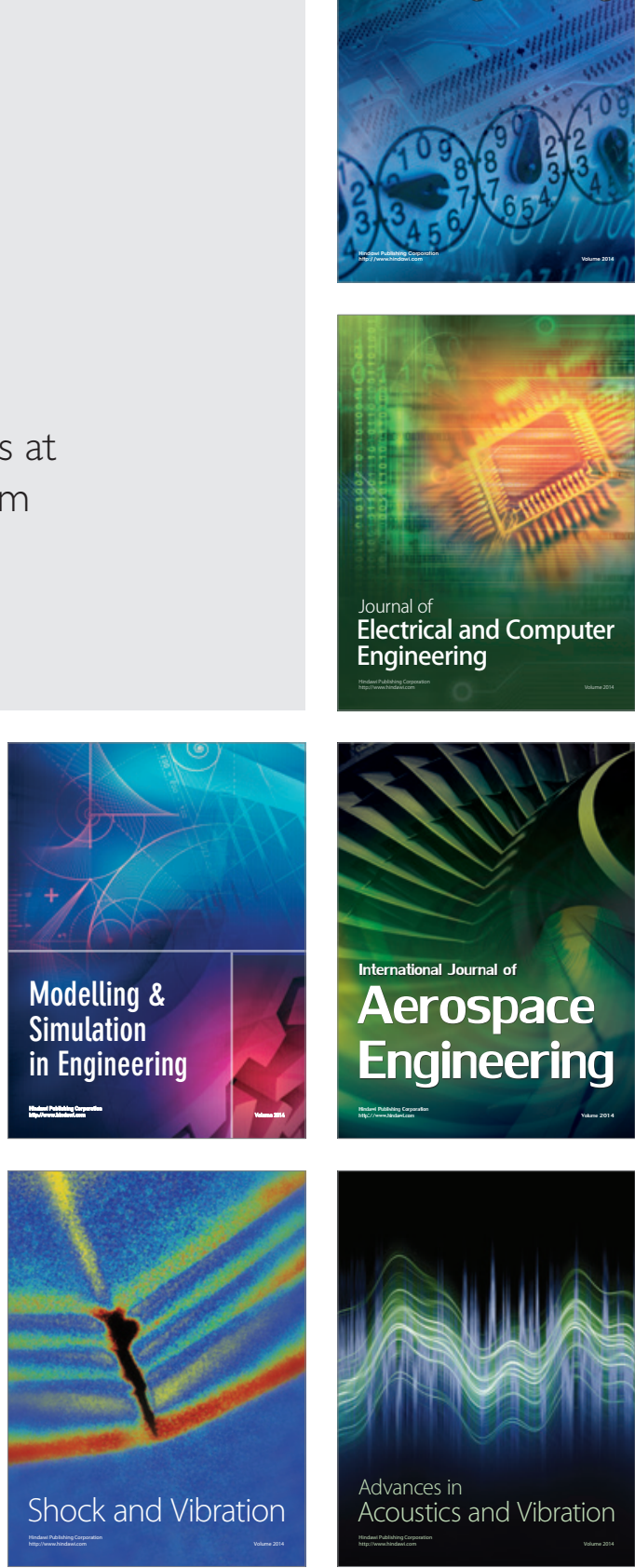